\title{
CMEARTICLE
}

\section{Clinics in diagnostic imaging (158)}

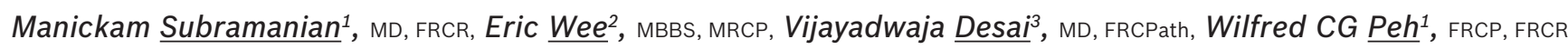

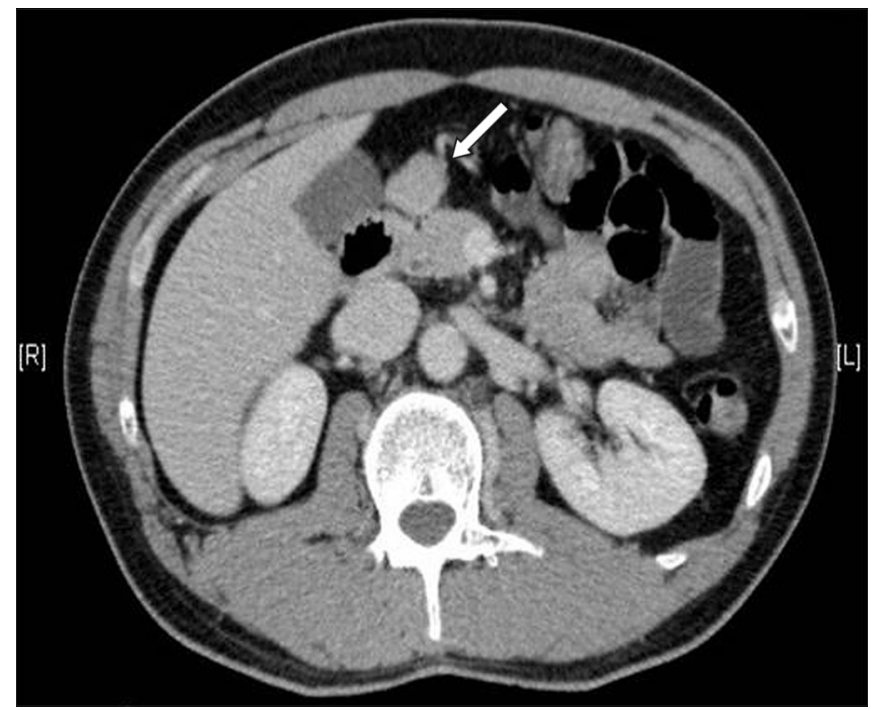

Fig. 1 Contrast-enhanced axial CT (portal venous phase) image of the upper abdomen.
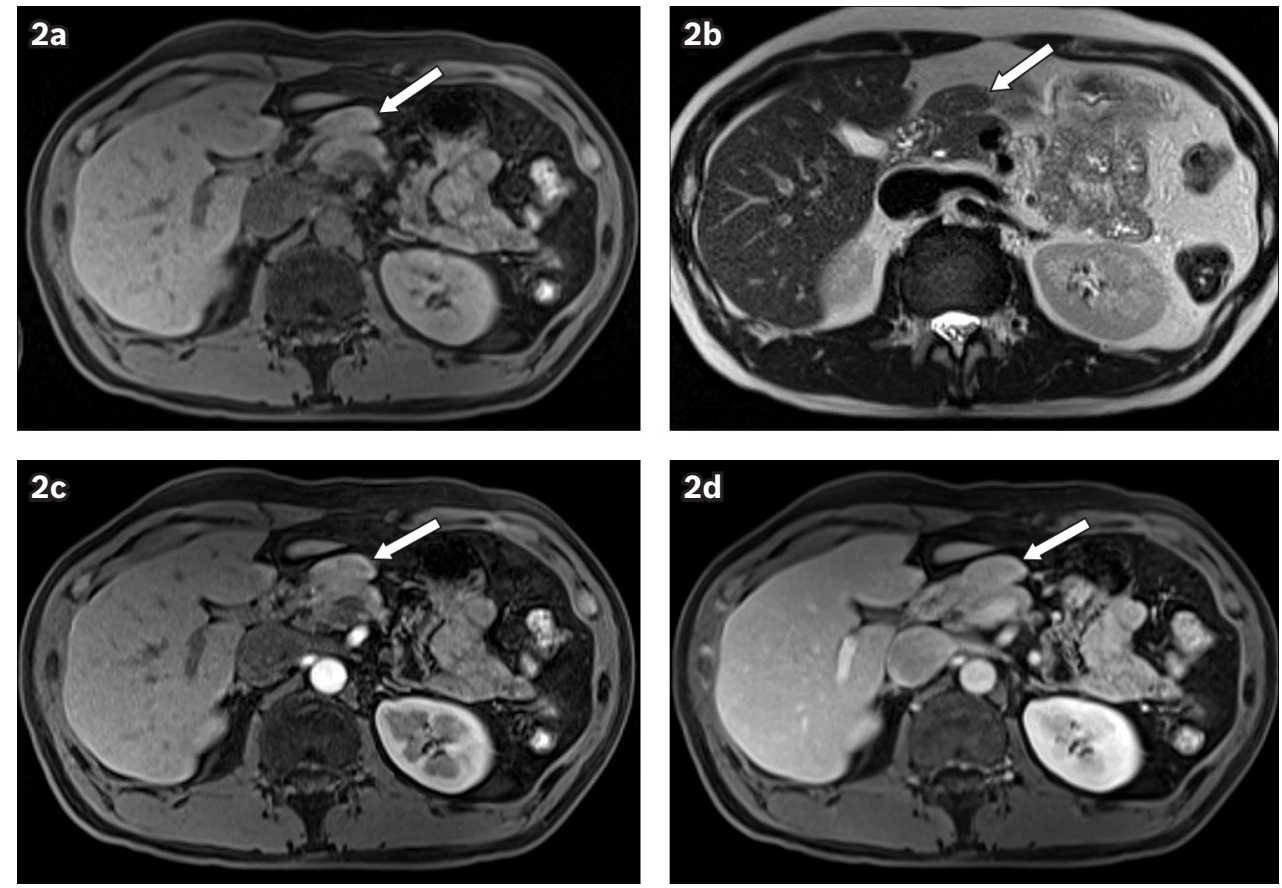

Fig. 2 Axial (a) T1-W and (b) T2-W MR images taken at the same level as Fig. 1. Contrast-enhanced (c) arterial and (d) portal venous phase T1-W MR images of the pancreas.

\section{CASE PRESENTATION}

A 46-year-old man without any significant past medical history presented to the emergency department with right loin tenderness after a road traffic accident. On physical examination, his abdomen was soft and non-tender. No palpable mass was felt. His routine blood investigations and chest radiograph were unremarkable. Focused assessment with sonography in trauma (FAST) was unremarkable and did not reveal free fluid. Contrastenhanced computed tomography (CT) (Fig.1) and magnetic resonance (MR) (Figs. 2a-d) imaging of the abdomen and pelvis were subsequently performed. What do these images show? What is the diagnosis? 

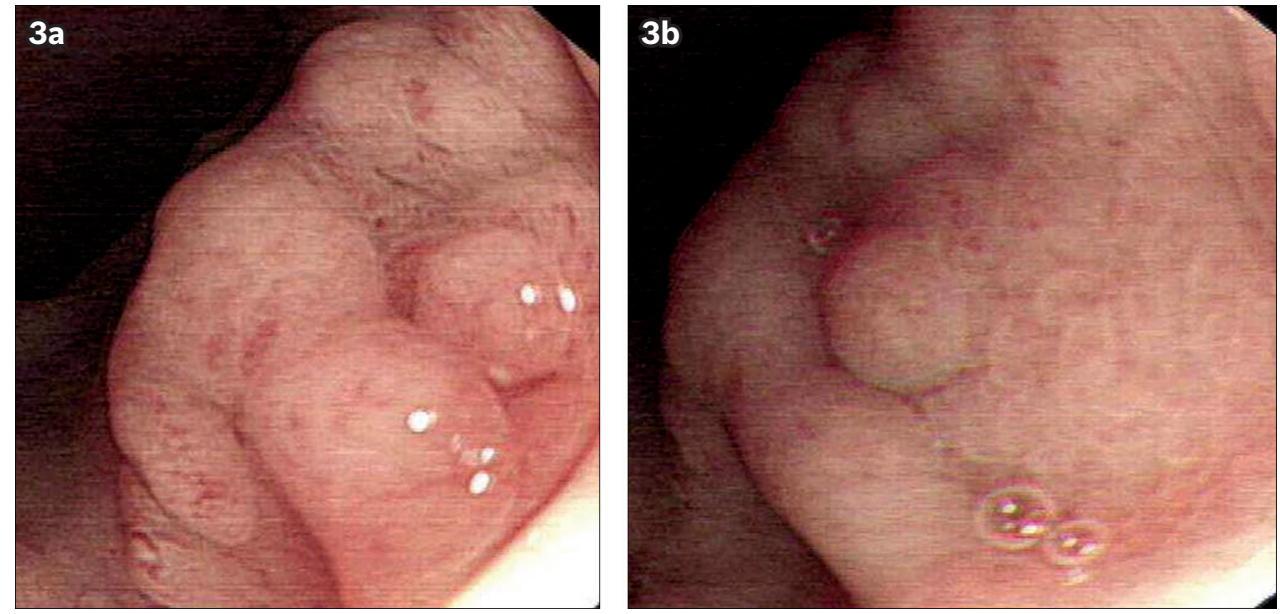

Fig. 3 (a-b) Gastroduodenoscopy images show a lobulated nodular submucosal lesion without central umbilication. The overlying mucosa is normal.
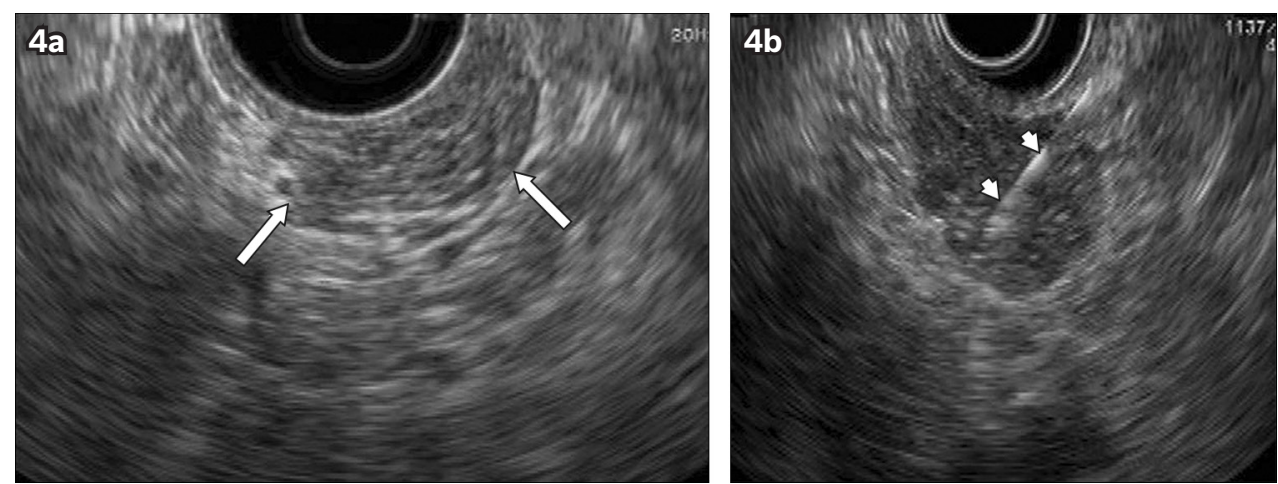

Fig. 4 EUS images show (a) a lobulated hypoechoic submucosal lesion (arrows) in the first part of the duodenum and (b) an echogenic biopsy needle (arrowheads) inserted into the lesion.

\section{IMAGE INTERPRETATION}

Contrast-enhanced axial CT image shows a well-defined, ovalshaped enhancing mass (arrow) with smooth borders in the wall of the first part of the duodenum (Fig. 1). The mass is separate from the pancreas. The pancreas appears normal in size, shape and attenuation. There is no dilatation of the main pancreatic duct. No free fluid or air is seen in the peritoneal cavity. The rest of the abdominal organs are normal. Axial T1-weighted and T2-weighted MR images show a well-defined, oval-shaped mass (arrow) with smooth borders in the wall of the first part of the duodenum. The mass has similar signal intensity to the pancreas (Figs. 2a-b). Contrast-enhanced axial arterial and portal venous phase T1-weighted MR images show that the mass (arrow) has similar enhancement to the pancreas (Figs. 2c-d).

\section{DIAGNOSIS}

Duodenal ectopic pancreas.

\section{CLINICAL COURSE}

The patient underwent oesophagogastroduodenoscopy, endoscopic ultrasonography (EUS) and fine-needle aspiration cytology (FNAC) of the mass lesion. Gastroduodenoscopy revealed a normal stomach and a smooth, lobulated nodular lesion deep to the mucosa in the posterior wall of the first part of the duodenum (Fig. 3). There was no mucosal lesion. EUS showed a well-defined, hypoechoic lesion in the submucosal layer of the posterior wall of the first part of the duodenum with some vascularity but without extension to the serosa (Fig. 4). There was no surrounding lymph node. The gastric wall was preserved and the pancreatic head was separate from the submucosal lesion. The pancreas appeared normal. No dilatation of the common bile duct and main pancreatic duct was noted. FNAC was performed with a 25G Boston needle. The patient tolerated the procedure well and was discharged on the same day. Cytological analysis of the aspirated material showed cellular smears with acinar structures identical to normal pancreatic tissue, consistent with ectopic pancreatic tissue (Fig. 5). There was no evidence of cellular atypia or malignancy. Since the patient was asymptomatic, surgical resection of the lesion was deemed unnecessary.

\section{DISCUSSION}

Ectopic or heterotopic pancreas is a relatively rare finding, with a reported incidence of $0.55 \%-13.70 \%$ at autopsy. ${ }^{(1)}$ It is defined as pancreatic tissue lying outside the normal anatomical location of the pancreas, without structural or vascular connection to the orthotopic pancreas. Ectopic pancreas is a congenital abnormality. More than $90 \%$ of cases occur in the upper gastrointestinal tract. The stomach, duodenum and the proximal jejunum are the most common sites. Other less commonly reported sites in the gastrointestinal tract are the ileum, Meckel's diverticulum, 


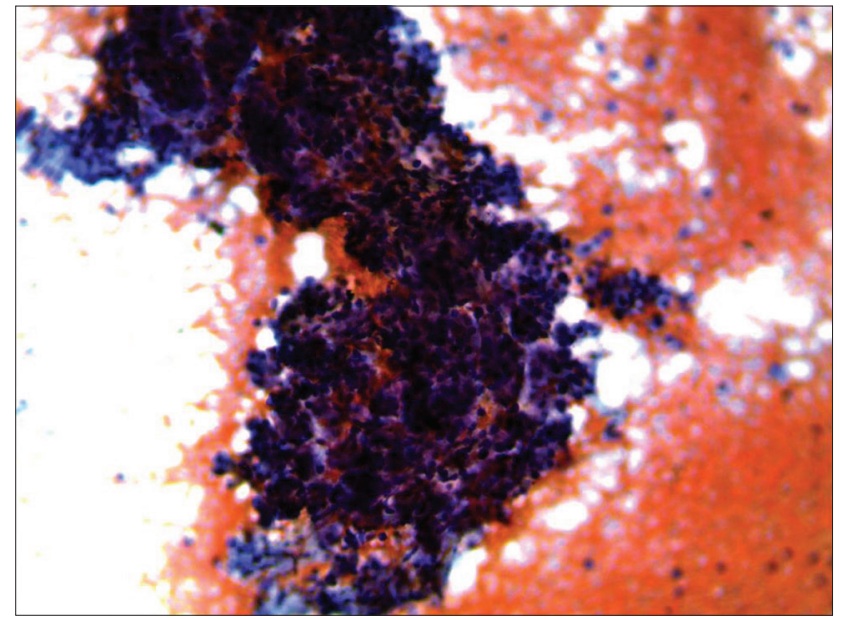

Fig. 5 Cytological photomicrograph shows pancreatic acinar structures (Papanicolaou's stain, × 20)

oesophagus, colon, mesentery and omentum. ${ }^{(2)}$ Ectopic pancreatic tissue has also been reported in the liver, gallbladder, spleen, common bile duct, retroperitoneum, umbilicus, lymph nodes, and in extra-abdominal locations such as the lungs and mediastinum..$^{(2-7)}$

By four to five weeks of gestation, the pancreas is formed by the ventral and dorsal buds of the endodermal lining of the duodenum. The dorsal bud grows more rapidly than the ventral bud. The dorsal bud forms the upper part of the head, body and tail of the pancreas, while the ventral bud forms the lower part of the head and uncinate process. The ventral bud rotates toward the dorsal bud and finally fuses with the dorsal bud. During the process of rotation, the ventral and dorsal buds of the pancreas are in close proximity to the stomach and duodenum. This may result in the incorporation of pancreatic primordial germ cells into the bowel loops, which may potentially develop into pancreatic tissue later on. Rarely, one of the buds may get detached and carried along the long axis of the bowel, thus explaining the site of ectopic pancreas remote from the normal anatomical location. ${ }^{(8)}$ Another theory of the origin of ectopic pancreas is the differentiation of totipotent endodermal cells into pancreatic tissue in the intestinal tract, as well as in extra-abdominal locations. This may explain the presence of pancreatic tissue in the lungs, mediastinum and retroperitoneum. ${ }^{(4,6,7)}$ Ectopic pancreas can be classified according to the Heinrich classification. Type I ectopic pancreas contains all the elements of normal pancreatic tissue such as acini, ducts and islet cells. Type II contains pancreatic acini and ducts without islet cells, while only pancreatic ducts are seen in Type III. ${ }^{(8)}$

Most cases of ectopic pancreas are incidentally diagnosed during gastroduodenoscopy, EUS or CT performed for other reasons, as in our case. However, patients with ectopic pancreas may be affected by the inflammatory or neoplastic process, similar to a normal pancreas. Patients with ectopic pancreas may present with complications such as bleeding, stricture, ulceration, pancreatitis, pseudocyst and malignant transformation. Most ectopic pancreas measure less than $2 \mathrm{~cm}$ in size, but can also be as large as $5 \mathrm{~cm} .{ }^{(2)}$ Ectopic pancreas occurring in the gastrointestinal tract is usually seen in the submucosal layer, making it difficult to differentiate from gastrointestinal stromal tumour (GIST). ${ }^{(9)}$
Imaging plays a crucial role in the diagnosis of ectopic pancreas. On barium meal, an ectopic pancreas appears as a smooth, extramucosal lesion with or without central umbilication in the greater curvature of the antropyloric region of the stomach or the proximal part of the duodenum. Central umbilication may represent a rudimentary pancreatic duct opening and is the key imaging feature of ectopic pancreas. ${ }^{(10)}$ On endoscopy, ectopic pancreas appears as a smooth, lobulated, nodular solid mass lesion deep to the mucosa with or without central duct opening (Fig. 3). EUS shows a well-defined, round or oval, hypoechoic submucosal lesion (Fig. 4a) with heteroechoic areas and some vascularity. ${ }^{(8)}$ EUS clearly demonstrates the five layers of the bowel wall and precisely localises the ectopic pancreas. It is also useful for guiding FNAC or biopsy (Fig. 4b).

On CT, ectopic pancreatic tissue is seen as a well-defined, round or oval enhancing lesion in the antropyloric wall of the stomach, or anywhere in the gastrointestinal tract. An example of another histologically proven ectopic pancreas in the proximal jejunal loop is shown in Fig. 6. Prominent mucosal enhancement overlying the lesion is a useful sign for differentiating ectopic pancreas from other submucosal lesions, as enhancement is due to repeated inflammation of the mucosa by underlying pancreatic tissue. ${ }^{(9)}$ Another useful sign is the degree of enhancement of ectopic pancreas, which is similar to that of normal pancreas in all phases (Fig. 6). MR imaging helps in the accurate diagnosis of ectopic pancreas, as it shows similar signal intensity to normal pancreas in all pulse sequences. Enhancement is also similar to the normal pancreas in all phases. ${ }^{(4)}$

Ectopic pancreas is often misdiagnosed, as it may resemble other submucosal lesions, such as GIST, leiomyoma, carcinoid, neuroendocrine tumours and intramural metastasis, on both imaging and during surgery. Fig. 7 shows an example of histologically proven GIST in the fundus of the stomach, which has similar imaging features as ectopic pancreas. It is difficult to establish an accurate diagnosis of ectopic pancreas by endoscopic biopsy or FNAC alone. Frozen section is routinely advised to confirm the diagnosis of ectopic pancreas, as radical surgery may potentially be avoided. ${ }^{(11)}$ Clinical significance of recognising asymptomatic ectopic pancreas is that it does not warrant further investigation. Management of incidentally detected, histologically confirmed ectopic pancreas in the stomach is disputable. ${ }^{(12)}$ When it is symptomatic, surgical resection is the treatment of choice. Laparoscopic resection of ectopic pancreas is a reliable and safe procedure. ${ }^{(13)}$

In summary, ectopic pancreas is a rare but well-known entity, both clinically and radiologically. The most common location of ectopic pancreas is the submucosal layer of the greater curvature of the antropyloric region of the stomach or the proximal part of the duodenum. Ectopic pancreas is usually asymptomatic and diagnosed incidentally. Most often, it is misdiagnosed as GIST or other submucosal lesions. Important clues that point to the diagnosis of ectopic pancreas include its similarities to CT attenuation, MR signal intensity and degree of enhancement with normal pancreas. Knowledge of the various locations and imaging appearance of ectopic pancreas are important for the reporting radiologist in making an accurate diagnosis. 

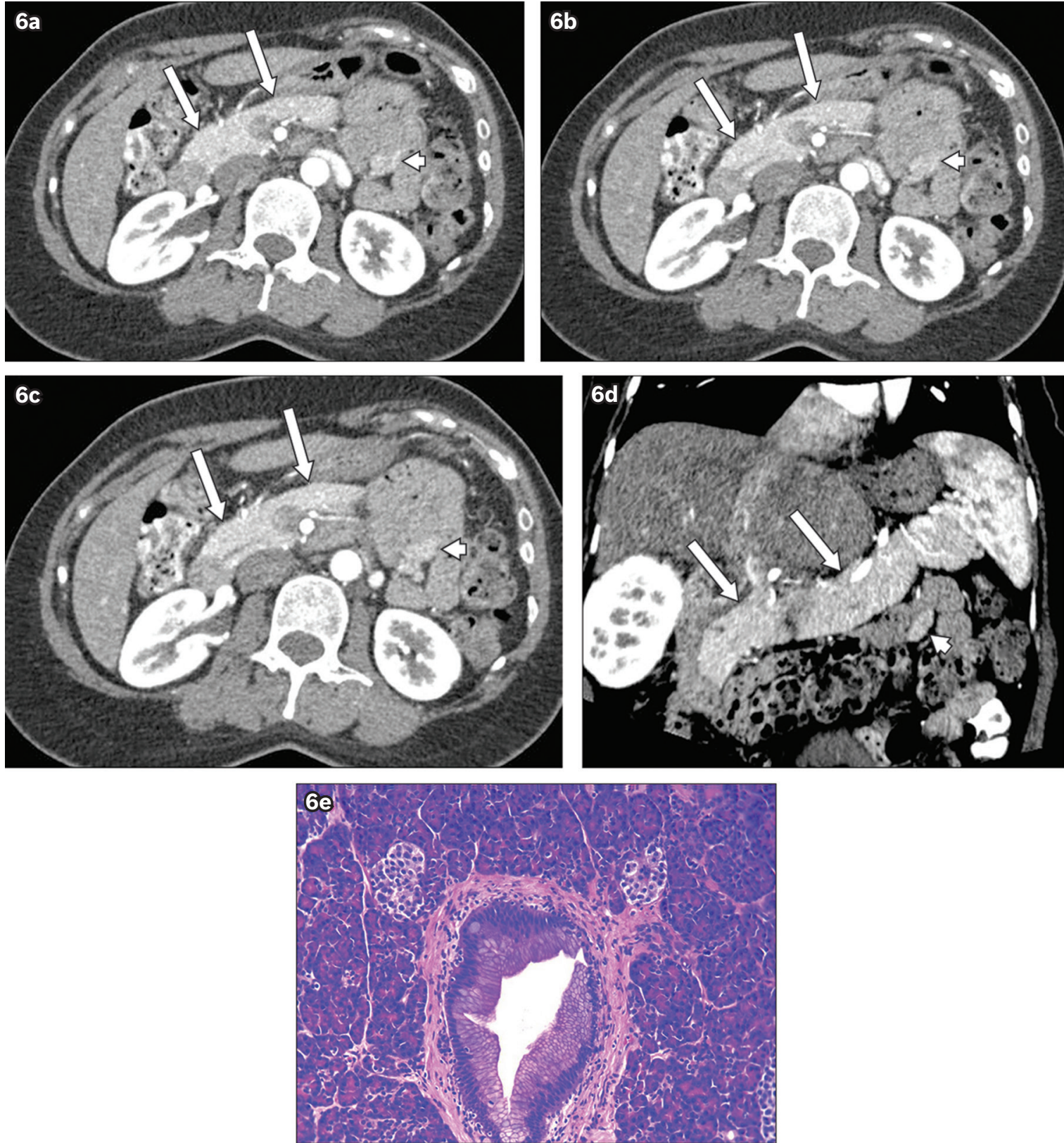

Fig. 6 Ectopic pancreas in a 52-year-old woman who presented with abdominal pain. (a-c) Contiguous axial and (d) coronal reconstructed arterial-phase CT images show an enhancing lesion (arrowhead) clustered within the proximal jejunal loop. The enhancement is identical to that of the normal pancreas (arrows). (e) Histological photomicrograph shows normal pancreatic acini, ducts and islet cells (Haematoxylin \& eosin, × 20).
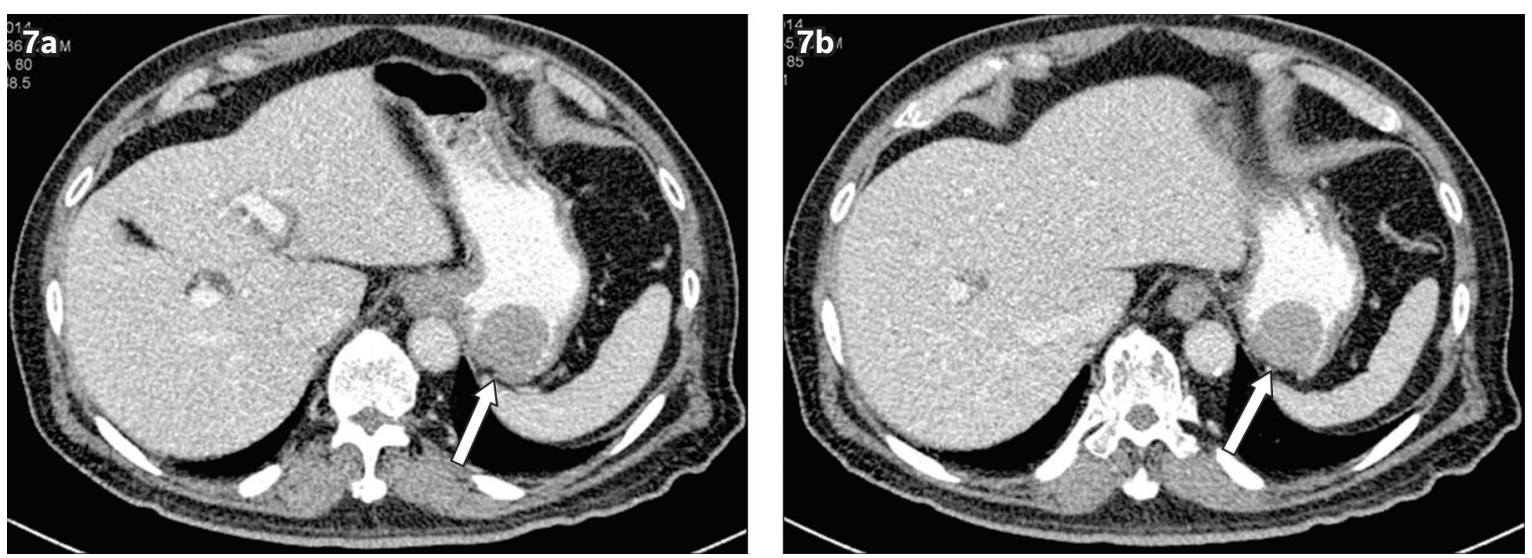

Fig. 7 Gastrointestinal stromal tumour (GIST) in a 77-year-old man who presented with pernicious anaemia. (a-b) Contiguous axial CT (portal venous phase) images of the abdomen shows a lesion with smooth borders in the fundus of the stomach (arrow). EUS guided biopsy showed a GIST. 
ABSTRACT A 46-year-old man presented with right loin tenderness following a road traffic accident. Computed tomography and magnetic resonance imaging showed a well-defined, smooth, enhancing oval lesion in the wall of the first part of the duodenum. The lesion was seen separately from the normal pancreas. It showed attenuation, intensity and enhancement similar to that of normal pancreas. Based on the imaging appearance, a diagnosis of ectopic pancreas was made. The patient underwent oesophagogastroduodenoscopy, endoscopic ultrasonography and fine-needle aspiration cytology of the lesion, which confirmed ectopic pancreatic tissue. Since the patient was asymptomatic, surgical resection was deferred. The embryologic origin, various locations, imaging appearance and clinical significance of ectopic pancreas are discussed.

Keywords: ectopic pancreas, pancreatic heterotopia, pancreatic mass, pancreatic pseudolesion

\section{REFERENCES}

1. Lai EC, Tompkins RK. Heterotopic pancreas. Review of a 26 year experience. Am J Surg 1986; 151:697-700.

2. Mortelé KJ, Rocha TC, Streeter JL, Taylor AJ. Multimodality imaging of pancreatic and biliary congenital anomalies. Radiographics 2006; 26:715-31.
3. Lee WT, Tseng HI, Lin JY, Tsai KB, Lu CC. Ectopic pancreatic tissue presenting as an umbilical mass in a newborn: a case report. Kaohsiung J Med Sci 2005; 21:84-7.

4. Lin LH, Ko SF, Huang CC, et al. Retroperitoneal ectopic pancreas: imaging findings. Br J Radiol 2009; 82:253-5.

5. Karahan OI, Kahriman G, Soyuer I, Artiş T, Comu NB. MR cholangiopancreatography findings of heterotopic pancreatic tissue in the distal common bile duct. Diagn Interv Radiology 2006; 12:180-2.

6. Marchevsky AM. Lung tumors derived from ectopic tissues. Semin Diagn Pathol 1995; 12:172-84

7. Wang W, Li K, Qin W, Sun H, Zhao C. Ectopic pancreas in mediastinum: report of 2 cases and review of the literature. J Thorac Imaging 2007; 22:256-8.

8. Zinkiewicz K, Juśkiewicz W, Zgodziński W, et al. Ectopic pancreas: endoscopic, ultrasound and radiological features. Folia Morphol (Warsz) 2003; 62:205-9.

9. Kim JY, Lee JM, Kim KW, et al. Ectopic pancreas: CT findings with emphasis on differentiation from small gastrointestinal stromal tumor and leiomyoma. Radiology 2009; 252:92-100.

10. $\mathrm{Yu}$ J, Turner MA, Fulcher AS, Halvorsen RA. Congenital anomalies and normal variants of the pancreaticobiliary tract and the pancreas in adults: part 2, pancreatic duct and pancreas. AJR Am J Roentgenol 2006; 187:1544-53.

11. Yuan Z, Chen J, Zheng Q, et al. Heterotopic pancreas in the gastrointestinal tract. World J Gastroenterol 2009; 15:3701-3.

12. Ormarsson OT, Gudmundsdottir I, Mårvik R. Diagnosis and treatment of gastric heterotopic pancreas. World J Surg 2006; 30:1682-9.

13. Gálvez-Valdovinos R, Mendoza-Rodríguez A, Coronado-Perez JH, Santillan EM, Funes-Rodríguez F. Laparoscopic treatment of heterotopic pancreas in the prepyloric region. J Minim Access Surg 2006; 2:224-6. 


\section{SINGAPORE MEDICAL COUNCIL CATEGORY 3B CME PROGRAMME} (Code SMJ 201412B)

Question 1. Regarding embryology of the pancreas:

(a) The pancreas is formed at around 15-16 weeks of gestation.

(b) It is formed by dorsal and ventral buds.

(c) The body and tail of the pancreas are formed by the dorsal bud.

(d) The ventral bud forms the uncinate process.

Question 2. Concerning ectopic pancreas:

(a) It is a congenital abnormality.

(b) It refers to pancreatic tissue lying outside the normal anatomical location with structural connection to the orthotopic pancreas.

(c) The most common site of occurrence is the upper gastrointestinal tract.

(d) It can occur outside the gastrointestinal tract.

Question 3. Regarding the presentation of ectopic pancreas:

(a) It is often diagnosed incidentally.

(b) It can present as pancreatitis.

(c) It is commonly seen in the serosa of gastrointestinal tract.

(d) It usually measures at least $5 \mathrm{~cm}$ in size.

Question 4. Concerning imaging features of ectopic pancreas:

(a) Extramucosal lesion with central umbilication in distal stomach or proximal duodenum is the classical finding on barium meal.

(b) On endoscopy, it appears as a lobulated solid mass deep to the mucosa with or without a central duct opening.

(c) On computed tomography, the degree of enhancement is similar to that of normal pancreas in all phases.

(d) On magnetic resonance imaging, ectopic pancreas shows similar signal intensity to normal pancreas in all pulse sequences.

Question 5. Concerning the diagnosis and management of ectopic pancreas:

(a) It may be misdiagnosed as gastrointestinal stromal tumour.

(b) Fine-needle aspiration cytology always establishes an accurate diagnosis.

(c) Surgical resection is the treatment of choice when symptomatic.

(d) Frozen section is routinely performed during surgery.

\section{Doctor's particulars:}

Name in full

MCR number

Email address

\section{SUBMISSION INSTRUCTIONS:}

(1) Log on at the SMJ website: http://www.sma.org.sg/publications/smjcurrentissue.aspx and select the appropriate set of questions. (2) Provide your name, email address and MCR number. (3) Select your answers and click "Submit".

\section{RESULTS:}

(1) Answers will be published in the SMJ February 2015 issue. (2) The MCR numbers of successful candidates will be posted online at the SMJ website by 30 January 2015 (3) Passing mark is $60 \%$. No mark will be deducted for incorrect answers. (4) The SMJ editorial office will submit the list of successful candidates to the Singapore Medical Council. (5) One CME point is awarded for successful candidates.

Deadline for submission: (December 2014 SMJ 3B CME programme): 12 noon, 23 January 2015. 